\title{
THE COEFFICIENTS OF MULTIVALENT CLOSE-TO-CONVEX FUNCTIONS
}

\section{ALBERT E. LIVINGSTON ${ }^{1}$}

1. Introduction. Let $S(p)$ denote the class of functions which are regular and star-like of order $p$ in the unit disk $E,|z|<1,[2],[4]$. A function

$$
f(z)=a_{1} z+a_{2} z^{2}+\cdots \quad(|z|<1)
$$

is a member of $S(p)$ if and only if there exists a positive number $\rho$ such that for $\rho<|z|<1$

$$
\operatorname{Re}\left(\frac{z f^{\prime}(z)}{f(z)}\right)>0, \quad \int_{0}^{2 \pi} \operatorname{Re}\left(\frac{z f^{\prime}(z)}{f(z)}\right) d \theta=2 p \pi .
$$

We shall say that a function $F(z)$ is in $\varkappa(p)$, if it is regular in $E$ and there exists a function $f(z)$ in $S(p)$ and a positive number $\rho$ such that for $\rho<|z|<1$

$$
\operatorname{Re}\left(\frac{z F^{\prime}(z)}{f(z)}\right)>0 .
$$

A function in $\varkappa(p)$ is at most $p$-valent in $E$ [5].

Goodman [1] has conjectured that if $f(z)=a_{1} z+a_{2} z^{2}+\cdots(|z|<1)$ is $p$-valent in $E$, then

$$
\left|a_{n}\right| \leqq \sum_{t=1}^{p} \frac{2 t(n+p) !}{(p+t) !(p-t) !(n-p-1) !\left(n^{2}-t^{2}\right)}\left|a_{t}\right| .
$$

Inequality (1.1) reduces to the well-known Bieberbach conjecture when $p=1$. The conjecture was proven by Goodman and Robertson [3] for a function in $S(p)$, in case all its coefficients are real and by Robertson [7], in case $a_{1}=a_{2}=\cdots=a_{p-2}=0$, the remaining coefficients being complex. The author [5] proved (1.1) for $n=p+1$ for functions in $\Re(p)$, no restrictions being made on the coefficients. In this paper, we will prove (1.1) for functions of the class $\Re(p)$ for the case $a_{1}=a_{2}=\cdots=a_{p-2}=0$, the remaining coefficients being complex. The case $p=2$ of our proof gives (1.1) for the entire class $\mathfrak{K}(2)$. Inequality (1.1) is known to be true for the class $\mathscr{K}(1)$ [6].

Received by the editors June 14, 1968.

${ }^{1}$ This research was supported by the University of Delaware Research Foundation. 
2. Preliminary lemmas. The following lemma is stated in greater generality than needed. We will make use only of the special case $s=1$.

Lemma 1. Let

$$
P(z)=c_{0}+c_{1} z+c_{2} z^{2}+\cdots \quad(|z|<1)
$$

be regular and satisfy the condition $\operatorname{Re}(P(z))>0$ in $E$, then for $n \geqq 2$ and $s \geqq 1$

$$
\left|\frac{c_{n}}{c_{0}}-\frac{c_{s} c_{n-s}}{c_{0}^{2}}\right| \leqq 2\left|\frac{\operatorname{Re} c_{0}}{c_{0}}\right| \leqq 2 .
$$

These inequalities are sharp for all $n$ and for all s, equality being attained for each $n$ and for each $s$ by the function

$$
P(z)=\left(\operatorname{Re} c_{0}\right)\left(\frac{1+z}{1-z}\right)+i \operatorname{Im} c_{0} \quad\left(\operatorname{Re} c_{0}>0\right) .
$$

Proof. Since any function $P(z)$, regular in $E$ and satisfying $\operatorname{Re} P(z)>0$ and $P(0)=c_{0}$ in $E$, is the limit of a sequence of functions of the form

$$
P(z)=\left(\operatorname{Re} c_{0}\right) \sum_{k=1}^{m} \lambda_{k} \frac{1+e^{i t_{k}}}{1-e^{i t_{z}}}+i \operatorname{Im} c_{0}
$$

where $\lambda_{k} \geqq 0,1 \leqq k \leqq m$, and $\sum_{k=1}^{m} \lambda_{k}=1$, we need only prove the lemma for functions of the form (2.1). For such functions,

$$
c_{n}=\left(\operatorname{Re} c_{0}\right) \sum_{k=1}^{m} 2 \lambda_{k} e^{i n t_{k}} \quad(n \geqq 1) .
$$

Therefore,

$$
\begin{aligned}
\mid \frac{c_{n}}{c_{0}} & -\frac{c_{s} c_{n-s}}{c_{0}{ }^{2}} \mid \\
& =\left|\frac{\operatorname{Re} c_{0}}{c_{0}} \sum_{k=1}^{m} 2 \lambda_{k} e^{i n t_{k}}-\left(\frac{\operatorname{Re} c_{0}}{c_{0}}\right)^{2} \sum_{k=1}^{m} 2 \lambda_{k} e^{i s t_{k}} \sum_{k=1}^{m} 2 \lambda_{k} e^{i(n-s) t_{k}}\right| \\
& =2\left|\frac{\operatorname{Re} c_{0}}{c_{0}}\right|\left|\sum_{k=1}^{m} \lambda_{k}\left(e^{i n t_{k}}-2 e^{i s t_{k}} Q\right)\right| \\
& =2\left|\frac{\operatorname{Re} c_{0}}{c_{0}}\right|\left|\sum_{k=1}^{m} \lambda_{k} B_{k}\right| \\
& \leqq 2\left|\frac{\operatorname{Re} c_{0}}{c_{0}}\right| \sum_{k=1}^{m} \lambda_{k}\left|B_{k}\right|
\end{aligned}
$$


where

$$
Q=\frac{\operatorname{Re} c_{0}}{c_{0}} \sum_{k=1}^{m} \lambda_{k} e^{i(n-s) t_{k}}
$$

and

$$
B_{k}=e^{i n t_{k}}-2 e^{i s t_{k}} Q, \quad 1 \leqq k \leqq m .
$$

Thus we need only prove that

$$
\sum_{k=1}^{m} \lambda_{k}\left|B_{k}\right| \leqq 1
$$

By the Schwarz inequality

$$
\left(\sum_{k=1}^{m} \lambda_{k}\left|B_{k}\right|\right)^{2} \leqq \sum_{k=1}^{m} \lambda_{k} \sum_{k=1}^{m} \lambda_{k}\left|B_{k}\right|^{2}=\sum_{k=1}^{m} \lambda_{k}\left|B_{k}\right|^{2} .
$$

Moreover, we have

$$
\begin{aligned}
\sum_{k=1}^{m} \lambda_{k}\left|B_{k}\right|^{2}= & +4|Q|^{2}-4 \operatorname{Re} \sum_{k=1}^{m} \lambda_{k} e^{-i(n-s) t_{k}} Q \\
\operatorname{Re} \sum_{k=1}^{m} \lambda_{k} e^{-i(n-s) t_{k}} Q & =\operatorname{Re} \sum_{k=1}^{m} \lambda_{k} e^{-i(n-s) t_{k}}\left(\frac{\operatorname{Re} c_{0}}{c_{0}} \sum_{q=1}^{m} \lambda_{q} e^{i(n-s) t_{q}}\right) \\
& =\operatorname{Re}\left(\frac{\operatorname{Re} c_{0}}{c_{0}} \sum_{q=1}^{m} \lambda_{q} e^{i(n-s) t_{q}} \sum_{k=1}^{m} \lambda_{k} e^{-i(n-s) t_{k}}\right) \\
& =\operatorname{Re}\left(\frac{\operatorname{Re} c_{0}}{c_{0}}\left|\sum_{q=1}^{m} \lambda_{q} e^{i(n-s) t_{q}}\right|^{2}\right) \\
& =\left(\frac{\operatorname{Re} c_{0}}{\left|c_{0}\right|}\right)^{2}\left|\sum_{q=1}^{m} \lambda_{q} e^{i(n-s) t_{q}}\right|^{2} \\
& =|Q|^{2} .
\end{aligned}
$$

From (2.3) and (2.4) we obtain

$$
\sum_{k=1}^{m} \lambda_{k}\left|B_{k}\right|^{2}=1
$$

This completes the proof of the lemma.

LEMMA 2. For $n \geqq p+2$ 


$$
\begin{aligned}
& \frac{2(p-1)(n+p) !}{(2 p-1) !(n-p-1) !\left(n^{2}-(p-1)^{2}\right)} \\
& +\frac{2(n+p) !}{(2 p-1) !(n-p-1) !\left(n^{2}-p^{2}\right)} \\
& \quad+\sum_{k=p+1}^{n-1}\left(\frac{4(p-1)(k+p) !}{(2 p-1) !(k-p-1) !\left(k^{2}-(p-1)^{2}\right)}\right. \\
& \left.\quad+\frac{4(k+p) !}{(2 p-1) !(k-p-1) !\left(k^{2}-p^{2}\right)}\right)+2 \\
& =\frac{2 n(n+p) !}{(2 p-1) !(n-p-1) !\left(n^{2}-(p-1)^{2}\right)}
\end{aligned}
$$

and

$$
\begin{aligned}
\frac{2 p(n+p) !}{(2 p) !(n-p-1) !\left(n^{2}-p^{2}\right)} & \\
& \quad+\sum_{k=p+1}^{n-1}\left(\frac{4 p(k+p) !}{(2 p) !(k-p-1) !\left(k^{2}-p^{2}\right)}\right)+2 \\
= & \frac{2 n(n+p) !}{(2 p) !(n-p-1) !\left(n^{2}-p^{2}\right)} .
\end{aligned}
$$

Proof. Denote the left side of (2.5) by $A_{n}$. Both sides of (2.5) are equal to $(8 / 3) p(p+1)(p+2)$ when $n=p+2$. Assume the equality is true for a particular value of $n \geqq p+2$. Replacing $n$ by $n+1$, the left side of $(2.5)$ can be written in the form

$$
\begin{gathered}
\frac{2(p-1)(n+p+1) !}{(2 p-1) !(n-p) !\left((n+1)^{2}-(p-1)^{2}\right)} \\
+\frac{2(n+p+1) !}{(2 p-1) !(n-p) !\left((n+1)^{2}-p^{2}\right)} \\
+\frac{2(p-1)(n+p) !}{(2 p-1) !(n-p-1) !\left(n^{2}-(p-1)^{2}\right)} \\
+\frac{2(n+p) !}{(2 p-1) !(n-p-1) !\left(n^{2}-p^{2}\right)}+A_{n} .
\end{gathered}
$$

Replacing $A_{n}$ in (2.7) by the right side of (2.5), combining the third and fifth terms and simplifying, (2.7) can be written in the form 


$$
\begin{aligned}
& \frac{2(p-1)(n+p+1) !}{(2 p-1) !(n-p) !\left((n+1)^{2}-(p-1)^{2}\right)} \\
& +\frac{2(n+p) !}{(2 p-1) !(n-p-1) !(n-p+1)} \\
& +\frac{2(n+p) !}{(2 p-1) !(n-p) !(n-p+1)}+\frac{2(n+p-1) !}{(2 p-1) !(n-p) !} \\
& =\frac{2(p-1)(n+p+1) !}{(2 p-1) !(n-p) !\left((n+1)^{2}-(p-1)^{2}\right)} \\
& +\frac{2(n+p) !}{(2 p-1) !(n-p-1) !(n-p+1)}\left[1+\frac{1}{n-p}\right] \\
& +\frac{2(n+p-1) !}{(2 p-1) !(n-p) !} \\
& =\frac{2(p-1)(n+p+1) !}{(2 p-1) !(n-p) !\left((n+1)^{2}-(p-1)^{2}\right)} \\
& +\frac{2(n+p) !}{(2 p-1) !(n-p) !}+\frac{2(n+p-1) !}{(2 p-1) !(n-p) !} \\
& =\frac{2(p-1)(n+p+1) !}{(2 p-1) !(n-p) !\left((n+1)^{2}-(p-1)^{2}\right)} \\
& +\frac{2(n+p+1)(n+p-1) !}{(2 p-1) !(n-p) !} \\
& =\frac{2(n+p+1) !}{(2 p-1) !(n-p) !\left((n+1)^{2}-(p-1)^{2}\right)} \\
& \cdot\left[(p-1)+\frac{(n+1)^{2}-(p-1)^{2}}{n+p}\right] \\
& =\frac{2(n+1)(n+p+1) !}{(2 p-1) !(n-p) !\left((n+1)^{2}-(p-1)^{2}\right)} \text {. }
\end{aligned}
$$

The last expression is the right side of equality (2.5) with $n$ replaced by $n+1$. This then completes the proof of equality (2.5) by induction.

Denote the left side of (2.6) by $B_{n}$. Both sides of (2.6) are equal to $(p+2)(2 p+1)$ when $n=p+2$. Assume (2.6) is true for some $n \geqq p+2$. Replacing $n$ by $n+1$, the left side of (2.6) can be written in the form 


$$
\frac{2 p(n+p+1) !}{(2 p) !(n-p) !\left((n+1)^{2}-p^{2}\right)}+\frac{2 p(n+p) !}{(2 p) !(n-p-1) !\left(n^{2}-p^{2}\right)}+B_{n}
$$

Replacing $B_{n}$ by the right side of (2.6), (2.9) can be written in the form

$$
\begin{aligned}
\frac{2 p(n+p+1) !}{(2 p) !(n-p) !\left((n+1)^{2}-p^{2}\right)}+\frac{2(n+p)(n+p) !}{(2 p) !(n-p-1) !(n-p)(n+p)} \\
=\frac{2 p(n+p+1) !}{(2 p) !(n-p) !\left((n+1)^{2}-p^{2}\right)}+\frac{2(n+p) !\left((n+1)^{2}-p^{2}\right)}{(2 p) !(n-p) !\left((n+1)^{2}-p^{2}\right)} \\
=\frac{2 p(n+p+1) !}{(2 p) !(n-p) !\left((n+1)^{2}-p^{2}\right)}\left[1+\frac{n+1-p}{p}\right] \\
=\frac{2(n+1)(n+p+1) !}{(2 p) !(n-p) !\left((n+1)^{2}-p^{2}\right)} .
\end{aligned}
$$

The last expression is the right side of $(2.6)$ with $n$ replaced by $(n+1)$. This then completes the proof of (2.6) by induction.

\section{Coefficient bounds.}

THEOREM. Let

$$
F(z)=\sum_{n=p-1}^{\infty} a_{n} z^{n} \quad(|z|<1)
$$

be a member of $\mathfrak{K}(p)$, then for $n \geqq p+1$

$$
\left|a_{n}\right| \leqq \sum_{t=p-1}^{p} \frac{2 t(n+p) !}{(p+t) !(n-p-1) !\left(n^{2}-t^{2}\right)}\left|a_{t}\right|
$$

and these inequalities are sharp in both the variables $\left|a_{p-1}\right|$ and $\left|a_{p}\right|$.

Proof. Since the author [5] has already proven (3.1) for $n=p+1$, we will assume in what follows that $n \geqq p+2$. We may assume without loss of generality that $F(z)$ is regular on $|z|=1$. Then [5, pp. 176177] there exists $f(z)=b_{p-1} z^{p-1}+b_{p} z^{p}+\cdots$ regular and star-like for $|z| \leqq 1$ such that $\operatorname{Re}\left(z F^{\prime}(z) / f(z)\right)>0(|z| \leqq 1)$. Let

$$
\frac{z F^{\prime}(z)}{f(z)}=P(z)=\sum_{n=0}^{\infty} c_{n} z^{n}
$$

Comparing coefficients we obtain for $n \geqq(p-1)$

$$
n a_{n}=\sum_{k=p-1}^{n} b_{k} c_{n-k}
$$


Considering (3.2) for the cases $n=p-1$ and $n=p$ and solving for $b_{p-1}$ and $b_{p}$ in terms of $a_{p-1}$ and $a_{p}$, we obtain

$$
b_{p-1}=\frac{(p-1) a_{p-1}}{c_{0}}, \quad b_{p}=\frac{p a_{p}}{c_{0}}-\frac{(p-1) a_{p-1} c_{1}}{c_{0}^{2}} .
$$

Combining (3.2) and (3.3), we obtain

$$
\begin{aligned}
n a_{n}= & c_{0} b_{n}+\sum_{k=p+1}^{n-1} b_{k} c_{n-k} \\
& +\left(\frac{c_{n-p+1}}{c_{0}}-\frac{c_{1} c_{n-p}}{c_{0}^{2}}\right)(p-1) a_{p-1}+\frac{p a_{p} c_{n-p}}{c_{0}}
\end{aligned}
$$

Using Lemma 1 with $s=1$ and the well-known inequality $\left|c_{k}\right| \leqq 2\left|c_{0}\right|$, we obtain

$$
\begin{aligned}
n\left|a_{n}\right| \leqq\left|c_{0}\right|\left|b_{n}\right| & +\sum_{k=p+1}^{n-1} 2\left|b_{k}\right|\left|c_{0}\right| \\
& +2(p-1)\left|a_{p-1}+2 p\right| a_{p} \mid .
\end{aligned}
$$

Since $f(z)$ is star-like of order $p$ in $E$ with $b_{1}=b_{2}=\cdots=b_{p-2}=0$, it follows from [7] that for $k \geqq p+1$

$$
\begin{aligned}
\left|b_{k}\right| \leqq & \frac{2(p-1)(k+p) !}{(2 p-1) !(k-p-1) !\left(k^{2}-(p-1)^{2}\right)}\left|b_{p-1}\right| \\
& +\frac{2 p(k+p) !}{(2 p) !(k-p-1) !\left(k^{2}-p^{2}\right)}\left|b_{p}\right| \\
\leqq & \frac{2(p-1)(k+p) !}{(2 p-1) !(k-p-1) !\left(k^{2}-(p-1)^{2}\right)} \frac{(p-1)\left|a_{p-1}\right|}{\left|c_{0}\right|} \\
& +\frac{2 p(k+p) !}{(2 p) !(k-p-1) !\left(k^{2}-p^{2}\right)} \\
& \cdot\left(\frac{p\left|a_{p}\right|}{\left|c_{0}\right|}+\frac{2(p-1)\left|a_{p-1}\right|}{\left|c_{0}\right|}\right) \\
= & \left(\frac{2(p-1)(k+p) !}{(2 p-1) !(k-p-1) !\left(k^{2}-(p-1)^{2}\right)}\right. \\
& +\left(\frac{2(k+p) !}{(2 p) !(k-p-1) !\left(k^{2}-p^{2}\right)}\right) \frac{p\left|a_{p}\right|}{\left|c_{0}\right|} \cdot
\end{aligned}
$$


Combining (3.5), (3.6) and Lemma 2

$$
\begin{aligned}
n\left|a_{n}\right| \leqq & \frac{2 n(p-1)(n+p) !}{(2 p-1) !(n-p-1) !\left(n^{2}-(p-1)^{2}\right)}\left|a_{p-1}\right| \\
& +\frac{2 n p(n+p) !}{(2 p) !(n-p-1) !\left(n^{2}-p^{2}\right)}\left|a_{p}\right| .
\end{aligned}
$$

Dividing the last inequality by $n$, we obtain (3.1). Inequality (3.1) is known to be sharp [3] for the class of functions in $S(p)$ whose power series have real coefficients, which is a subclass of $\mathscr{K}(p)$.

\section{REFERENCES}

1. A. W. Goodman, On some determinants related to $p$-valent functions, Trans. Amer. Math. Soc. 63 (1948), 175-192.

2. - On the Schwarz-Christoffel transformation and p-valent functions, Trans. Amer. Math. Soc. 68 (1950), 204-223.

3. A. W. Goodman and M. S. Robertson, A class of multivalent functions, Trans. Amer. Math. Soc. 70 (1951), 127-136.

4. J. A. Hummel, Multivalent starlike functions, J. Analyse Math. 18 (1967), 133160.

5. Albert E. Livingston, p-valent close-to-convex functions, Trans. Amer. Math. Soc. 115 (1965), 161-179.

6. Maxwell O. Reade, The coefficients of close-to-convex functions, Duke Math. J. 23 (1950), 459-462.

7. M. S. Robertson, Multivalently star-like functions, Duke Math. J. 20 (1953), 539-550.

UNIVERSITY OF Delaware 